\title{
Recent tau-targeted clinical strategies for the treatment of Alzheimer's disease
}

\author{
Ha E Lee1,2, Dami Lim¹,3, Jae Y Lee ${ }^{3}$, Sang M Lim**,1,2 \& Ae N Pae*,1,2,3 \\ ${ }^{1}$ Convergence Research Center for Diagnosis, Treatment \& Care System of Dementia, Korea Institute of Science \& Technology, \\ Seoul 02792, Republic of Korea \\ ${ }^{2}$ Division of Bio-Medical Science \& Technology, KIST School, Korea University of Science \& Technology, Seoul 02792, Republic of \\ Korea \\ ${ }^{3}$ KHU-KIST Department of Converging Science \& Technology, Kyung Hee University, Seoul 02447, Republic of Korea \\ *Author for correspondence: anpae@kist.re.kr \\ **Author for correspondence: smlim28@kist.re.kr
}

\begin{abstract}
"A variety of tau-directed strategies for the treatment of Alzheimer's disease are being investigated in clinical trials and getting more attention after several amyloid $\beta$-targeting drug candidates failed to present promising clinical benefits"
\end{abstract}

First draft submitted: 14 May 2019; Accepted for publication: 31 May 2019; Published online: 13 September 2019

Keywords: acetylation • aggregation $\bullet$ Alzheimer's disease $\bullet$ deglycosylation $\bullet$ immunotherapy $\bullet$ tau

Recently terminated Phase III clinical trials of Aducanumab and Crenezumab have led researchers to further question the amyloid hypothesis for the onset of Alzheimer's disease (AD). These antibodies once had been considered as one of the most promising agents to be approved for AD. Due to the failure of clinical trials of these anti-amyloid $\beta$ antibodies along with the unsuccessful studies of BACE1 inhibitors such as Lanabecestat and Verubecestat, people increasingly believe that modulation of amyloid $\beta$ alone might not be enough to improve memory and cognitive function of $\mathrm{AD}$ patients. Many people postulate that clinical trials have failed because of the late administration of candidate drugs based on PET studies suggesting that amyloid $\beta$ plaques are accumulated decades before clinical symptoms are manifested [1]. Nevertheless, it became apparent that the amyloid hypothesis might explain only part of the complex pathologies of Alzheimer's diseases [2]. The rapidly aging global population urgently necessitates development of disease modifying therapies for $\mathrm{AD}$, thus scientists propose that it is 'time to cast a wider net' [3] and to search for alternative promising targets in addition to amyloid $\beta$.

\section{Tau-targeted therapeutic strategies}

Tau protein has been largely regarded as another promising target for $\mathrm{AD}$ because paired helical filaments (PHFs) and neurofibrillary tangles (NFTs) that are mainly composed of various tau species have been typically observed in brains of $\mathrm{AD}$ patients. Moreover, the severity of tau pathology is more closely correlated with the progression of cognitive decline of $\mathrm{AD}$ patients than amyloid $\beta$ [4]. Abnormal phosphorylation of tau followed by dissociation from microtubules has been considered as the key event initiating tau pathologies in $\mathrm{AD}$ and other tau-related diseases (tauopathies) [5]. In this regard, extensive efforts have been made to modulate (de)phosphorylation of tau for Alzheimer's disease as demonstrated by clinical trials with GSK3 $\beta$ inhibitors (Tideglusib and lithium) and PP2A activator (sodium selenate).

Recently, it is increasingly admitted that tau oligomers might be the most toxic tau species resulting in neurotoxicity and neurodegeneration in $\mathrm{AD}[6,7]$. Dissociation of hyperphosphorylated tau from microtubules produces greater amount of cytosolic tau that is prone to self-aggregation. Hence, inhibition of the process to produce toxic tau oligomers has been considered an effective therapeutic approach for AD. LMTX (TRx0237) has been extensively investigated as a tau aggregation inhibitor. Clear clinical benefits were not observed in two Phase III clinical trials (NCT01689246, NCT01689233). Interestingly, LMTX has shown initial promise as a monotherapy for mild-to-moderate $\mathrm{AD}$ patients who have not taken other $\mathrm{AD}$ drugs. Therefore, another Phase II/III clinical 
trial of LMTX was launched to further investigate safety and efficacy as a monotherapy to early AD patients (NCT03446001).

In addition to these strategies, relatively new approaches based on tau have been currently investigated as well: inhibition of tau acetylation and deglycosylation, tau immunotherapy and reduction of tau level. First, tau was found to be highly acetylated in brains of $\mathrm{AD}$ patients [8]. Tau acetylation is mainly mediated by p300 and CBP and the reverse deacetylation is processed by SIRT1 [8]. Acetylation occurs at lysine residues of tau that can prevent at least partially polyubiquitination of tau. In turn, proteasome-mediated tau degradation can be impaired facilitating accumulation of both cytosolic and hyperphosphorylated tau followed by enhanced tau oligomerization [8]. In addition, acetylation of tau disrupts the association between tau and microtubules by neutralizing the positive charge of lysine residues of tau, again increasing the amount of free tau species. For instance, overexpression of an acetylated mimic of K280 of human tau in Drosophila transgenic models substantially promoted phosphorylation at S262 and exacerbated photoreceptor neurodegeneration [9]. Similarly, introduction of the K174Q mutant human tau to C57BL/6 mice slowed tau turnover and aggravated neurodegeneration and behavioral impairment [10]. Pharmacological inhibition of p300 with salsalate in PS19 mice reduced the level of acetylated tau at K174 subsequently ameliorating hippocampal atrophy and memory deficits [10], which suggests perturbing tau acetylation could be an effective therapeutic strategy for $\mathrm{AD}$ and tauopathies. Thus, pilot futility clinical trial of salsalate (NCT02422485) was launched to confirm the preliminary efficacy, safety and tolerability with progressive supranuclear palsy (PSP) patients. Furthermore, a Phase Ib clinical trial (NCT03277573) is currently being tested to assess safety and tolerability of salsalate in prodromal to mild $\mathrm{AD}$ patients.

It is frequently found from FDG PET studies that brain glucose uptake and metabolism are compromised in brains of AD patients [11]. In this perspective, $O$-GlcNAcylation of tau is substantially reduced in AD patients as well. Tau $O$-GlcNAcylation has received attention because it is negatively correlated with tau phosphorylation [12]. Furthermore, it was shown that incorporation of $O$-GlcNAc to tau slowed down tau aggregation [13] suggesting enhancing tau $O$-GlcNAcylation might be a promising approach to reduce tau pathology and neurodegeneration. Tau $O$-glycosylation can be accessed by inhibition of OGA that is responsible for removing $O$-GlcNAc from tau. It was reported that chronic treatment of an OGA inhibitor, Thiamet G was shown to decrease pathological tau species in the brain and total tau in a tau transgenic mouse model [14]. Encouraging results of Phase I clinical trials with selective OGA inhibitors, MK-8719 prompted US FDA to grant MK-8719 orphan drug status for PSP. In addition, another OGA inhibitor ASN120290 exhibited promising Phase I clinical trial results that led to receiving orphan drug designation for PSP by FDA. Currently, neuroimaging trial for ASN120290 is initiated to confirm target engagement of this agent in human.

Intracellular tau oligomerization once rendered researchers a little skeptical of the feasibility of target engagement by tau immunization. However, since promising therapeutic effects have been demonstrated in tau transgenic mice with either active [15] or passive immunization [16] based on tau, there have been a surge to find effective tau immunization approaches targeting a variety of tau epitopes. Still the precise mechanism how tau-specific antibodies can penetrate into neurons remains to be elucidated, but $\mathrm{Fc} \gamma$ receptors or bulk-mediated endocytosis are considered as most probable ways [17]. Once tau-targeting antibodies are taken up by neurons, it is believed that they promote intracellular clearance and sequestration of pathological tau. Another hypothesis of tau immunotherapy is that targeting extracellular pathological tau would produce clinical benefits by inhibition of the cell-to-cell transmission and consequently the spread of tau pathology. It was shown that intracellular tau oligomers were secreted and subsequently taken up by neighboring cells triggering tau oligomerization in receiving cells [18]. In this way, tau-targeting antibodies do not necessarily need to penetrate into neurons to exert their effects. Recently, it was demonstrated that intraperitoneal dosing of ABBV-8E12 to P301S mice substantially attenuated tau pathology in the hippocampus and prevented brain atrophy [19]. Currently, most passive tau immunotherapy under clinical trials are designed to target extracellular tau species. There are three passive tau antibodies in Phase II clinical trials. BIIB092 and ABBV-8E12, both of which target the amino-terminus of tau presented encouraging Phase I trial results. As a result, Phase II clinical trials have been initiated with patients with PSP (NCT03068468, NCT03391765) and mild AD (NCT03352557, NCT03712787) with these two antibodies, respectively. Another passive tau immunotherapy RO7105705 is also being tested in a Phase II clinical trial for prodromal to mild AD patients (NCT03289143). In addition to these therapeutic agents, AADvac1 and ACI-35 are investigated in clinical trials for $\mathrm{AD}$ patients as active tau immunotherapy.

Another promising strategy is to lower the level of tau monomer thereby depleting substrates for tau aggregation or shifting the equilibrium to tau depolymerization. Although whether endogenous tau knockdown is not detrimental 
in vivo is yet to be elucidated further, recent confirmation of the safety profile of antisense oligonucleotide (ASO)based clinical trials and validation of efficacy and safety in nonhuman primates [20] prompted Phase I/II clinical trials of IONIS-MAPT $\mathrm{RX}$, an ASO-targeting tau expression (NCT03186989).

\section{Future perspective}

A variety of tau-directed strategies for the treatment of Alzheimer's disease are being investigated in clinical trials and getting more attention after several amyloid $\beta$-targeting drug candidates failed to present promising clinical benefits. Nevertheless, in order to develop disease-modifying tau-based therapeutics, researchers should be cautious about potential caveats of each tau-centric approach. For example, complete inhibition of p300 to block tau acetylation may provoke serious adverse effects because of multi-functional roles of p300. Also, it should be carefully investigated whether chronic treatment of OGA inhibitors would lead to improving cognitive deficits even though it was shown to exhibit little effect on tau phosphorylation. Moreover, it is a matter of primary interest that perturbation of the cell-to-cell tau transmission would eventually be translated into promising clinical benefits in human. Further extensive integrated research on tau pathology and related pathobiology upon treating tau-directed drug candidates is required to circumvent these potential obstacles. Careful design of clinical trials such as decision on appropriate dosing amount and exploration of possible combination strategy will help to maximize therapeutic potentials of each tau-directed strategy as well. Last, thorough analysis of clinical trials with tau-targeted drug candidates as well as other $\mathrm{AD}$ trials will eventually help to develop disease-modifying therapeutics for $\mathrm{AD}$.

\section{Financial \& competing interests disclosure}

This work was supported by the National Research Council of Science \& Technology grant by the Korea government (MSIP) (no. CRC-15-04-KIST). The authors have no other relevant affiliations or financial involvement with any organization or entity with a financial interest in or financial conflict with the subject matter or materials discussed in the manuscript apart from those disclosed.

No writing assistance was utilized in the production of this manuscript.

\section{References}

1. Mehta D, Jackson R, Paul G, Shi J, Sabbagh M. Why do trials for Alzheimer's disease drugs keep failing? A discontinued drug perspective for 2010-2015. Expert Opin. Investig. Drugs 26(6), 735-739 (2017).

2. Chetelat G. Alzheimer disease: abeta-independent processes-rethinking preclinical AD. Nat. Rev. Neurol. 9(3), 123-124 (2013).

3. Makin S. The amyloid hypothesis on trial. Nature 559(7715), S4-S7 (2018).

4. Bejanin A, Schonhaut DR, La Joie R et al. Tau pathology and neurodegeneration contribute to cognitive impairment in Alzheimer's disease. Brain 140(12), 3286-3300 (2017).

5. Iqbal K, Liu F, Gong CX. Tau and neurodegenerative disease: the story so far. Nat. Rev. Neurol. 12(1), 15-27 (2016).

6. Shafiei SS, Guerrero-Munoz MJ, Castillo-Carranza DL. Tau oligomers: cytotoxicity, propagation, and mitochondrial damage. Front. Aging Neurosci. 9, 83 (2017).

7. Cardenas-Aguayo Mdel C, Gomez-Virgilio L, Derosa S, Meraz-Rios MA. The role of tau oligomers in the onset of Alzheimer's disease neuropathology. ACS Chem. Neurosci. 5(12), 1178-1191 (2014).

8. Min SW, Cho SH, Zhou Y et al. Acetylation of tau inhibits its degradation and contributes to tauopathy. Neuron 67(6), 953-966 (2010).

9. Gorsky MK, Burnouf S, Dols J, Mandelkow E, Partridge L. Acetylation mimic of lysine 280 exacerbates human Tau neurotoxicity in vivo. Sci. Rep. 6, 22685 (2016).

10. Min SW, Chen X, Tracy TE et al. Critical role of acetylation in tau-mediated neurodegeneration and cognitive deficits. Nat. Med. 21(10), 1154-1162 (2015).

11. Mosconi L, Pupi A, De Leon MJ. Brain glucose hypometabolism and oxidative stress in preclinical Alzheimer's disease. Ann. NY Acad. Sci. 1147, 180-195 (2008).

12. Liu F, Shi J, Tanimukai H et al. Reduced O-GlcNAcylation links lower brain glucose metabolism and tau pathology in Alzheimer's disease. Brain 132(Pt 7), 1820-1832 (2009).

13. Yuzwa SA, Cheung AH, Okon M, Mcintosh LP, Vocadlo DJ. O-GlcNAc modification of tau directly inhibits its aggregation without perturbing the conformational properties of tau monomers. J. Mol. Biol. 426(8), 1736-1752 (2014).

14. Hastings NB, Wang X, Song L et al. Inhibition of O-GlcNAcase leads to elevation of O-GlcNAc tau and reduction of tauopathy and cerebrospinal fluid tau in rTg4510 mice. Mol. Neurodegener. 12(1), 39 (2017).

15. Asuni AA, Boutajangout A, Quartermain D, Sigurdsson EM. Immunotherapy targeting pathological tau conformers in a tangle mouse model reduces brain pathology with associated functional improvements. J. Neurosci. 27(34), 9115-9129 (2007). 
16. Boutajangout A, Ingadottir J, Davies P, Sigurdsson EM Passive tau immunotherapy diminishes functional decline and clears tau aggregates in a mouse model of tauopathy. Alzheimer's Dementia 6(4), S578 (2010).

17. Congdon EE, Gu J, Sait HB, Sigurdsson EM. Antibody uptake into neurons occurs primarily via clathrin-dependent Fc $\gamma$ receptor endocytosis and is a prerequisite for acute tau protein clearance. J. Biol. Chem. 288(49), 35452-35465 (2013).

18. Kfoury N, Holmes BB, Jiang H, Holtzman DM, Diamond MI. Trans-cellular propagation of Tau aggregation by fibrillar species. J. Biol. Chem. 287(23), 19440-19451 (2012).

19. West T, Hu Y, Verghese P et al. Preclinical and clinical development of ABBV-8E12, a humanized anti-tau antibody, for treatment of Alzheimer's disease and other tauopathies. J. Prev. Alzheimers Dis. 4(04), 236-241 (2017).

20. Mignon L, Kordasiewicz H, Lane R et al. Design of the first-in-human study of IONIS-MAPT $\mathrm{RX}$, a Tau-lowering antisense oligonucleotide, in patients with Alzheimer disease. Neurology 90(15 Suppl.), S2.006 (2018). 